\title{
Reflets
}

Revue d'intervention sociale et communautaire

\section{Les dilemmes éthiques en contexte de stage international : proposition d'un modèle de délibération qui intègre l'analyse intersectionnelle}

\author{
Hélène Albert, Isabel Lanteigne et Lise Savoie
}

Volume 20, numéro 1, printemps 2014

La formation pratique : allier milieux de pratique et milieux d'enseignement

URI : https://id.erudit.org/iderudit/1025795ar

DOI : https://doi.org/10.7202/1025795ar

Aller au sommaire du numéro

Éditeur(s)

Reflets, Revue d'intervention sociale et communautaire

ISSN

1203-4576 (imprimé)

1712-8498 (numérique)

Découvrir la revue

Citer cet article

Albert, H., Lanteigne, I. \& Savoie, L. (2014). Les dilemmes éthiques en contexte de stage international : proposition d'un modèle de délibération qui intègre l'analyse intersectionnelle. Reflets, 20(1), 76-105.

https://doi.org/10.7202/1025795ar
Résumé de l'article

La formation pratique dans un contexte international est de plus en plus recherchée par les stagiaires en travail social. L'École de travail social de l'Université de Moncton possède une tradition de plus de vingt-cinq ans à cet effet. Les stages internationaux suscitent des dilemmes éthiques nécessitant un encadrement qui assure une préparation et un accompagnement soucieux de la complexité des enjeux rencontrés. Le présent article propose un modèle de délibération éthique qui intègre les dimensions théoriques de l'analyse intersectionnelle en vue de l'accompagnement de stagiaires. Le modèle présenté cherche à contribuer à la réflexivité de façon à éviter des pratiques oppressives.
Tous droits réservés (C) Reflets, Revue d’intervention sociale et communautaire, 2014
Ce document est protégé par la loi sur le droit d'auteur. L'utilisation des services d'Érudit (y compris la reproduction) est assujettie à sa politique d'utilisation que vous pouvez consulter en ligne. 


\section{Les dilemmes éthiques en contexte de stage international : proposition d'un modèle de délibération qui intègre l'analyse intersectionnelle}

Hélène Albert

Professeure, École de travail social, Université de Moncton

Isabel Lanteigne

Professeure, Ecole de travail social, Université de Moncton

Lise Savoie

Professeure, École de travail social, Université de Moncton

\section{Résumé}

La formation pratique dans un contexte international est de plus en plus recherchée par les stagiaires en travail social. L'École de travail social de l'Université de Moncton possède une tradition de plus de vingt-cinq ans à cet effet. Les stages internationaux suscitent des dilemmes éthiques nécessitant un encadrement qui assure une préparation et un accompagnement soucieux de la complexité des enjeux rencontrés. Le présent article propose un modèle de délibération éthique qui intègre les dimensions théoriques de l'analyse intersectionnelle en vue de l'accompagnement de stagiaires. Le modèle présenté cherche à contribuer à la réflexivité de façon à éviter des pratiques oppressives.

Mots clés : Délibération éthique, stage international, analyse intersectionnelle, encadrement, formation pratique, réflexivité, pratiques antioppressives, systèmes d'oppression et de privilège, identités. 


\section{Abstract}

International field placements are gaining popularity among social work students who are requesting more and more placements abroad. The School of Social Work at the Université de Moncton has sent students abroad for the past twenty-five years. Such placements require adequate preparation and supervision to assist students with complex realities and ethical dilemmas encountered in the host country. The article presents a model developed to help students with ethical deliberation. An intersectional analysis is at the core of the model in order to promote reflexivity and anti-oppressive practices.

Key words : Ethical deliberation, international field placement, intersectional analysis, supervision, reflexivity, antioppressive practice, systems of oppression and privilege, identities.

Depuis plus de 25 ans, l'École de travail social (ÉTS) de l'Université de Moncton encourage les stages de formation pratique à l'international, soit en Afrique du Nord ou en Europe. L'intérêt du corps professoral pour les questions internationales, l'engagement de quelques membres à promouvoir des échanges internationaux et le désir des stagiaires de vivre une expérience internationale font en sorte que de nombreux stages se déroulent dans un tel contexte. Cette expérience fournit l'occasion aux stagiaires de développer et d'adapter les savoirs, savoir-être et savoir-faire interculturels. Selon la Fédération internationale des travailleurs sociaux et l'Association internationale des écoles de travail social (2004), les stagiaires doivent recevoir une formation pratique de qualité et qui soit à jour, entre autres exemples, sur les débats éthiques qui dépassent les frontières. De plus, l'Association canadienne pour la formation en travail social (2013, p. 12) exige que les étudiantes et les étudiants « connaissent les codes d`éthique du travail social associés à divers rôles et activités professionnels et à différents contextes institutionnels ». Cependant, les stages internationaux imposent un encadrement rigoureux quant à leur sélection et quant à la préparation, à la supervision et au debriefing des stagiaires qui séjournent à l'étranger. Des enjeux éthiques 
marquent souvent chacune de ces étapes. Par ailleurs, la dimension interculturelle d'une telle expérience offre un contexte privilégié pour apprécier la pertinence de l'analyse intersectionnelle afin de mieux saisir la complexité des situations rencontrées en stage. Le présent article propose un modèle de délibération éthique qui intègre les dimensions théoriques de l'analyse intersectionnelle en vue de l'accompagnement de stagiaires dans un contexte international. Nous y traitons d'abord des enjeux qui se présentent lors de stages internationaux et nous explorons les concepts d'intersectionnalité et de processus de délibération éthique. Puis, nous proposons un modèle appliqué à un dilemme éthique.

\section{Des enjeux rencontrés lors de stages internationaux}

La littérature portant sur les stages internationaux aborde de nombreux défis qui se posent aux stagiaires. Engstrom et Jones (2007) se penchent sur les apprentissages que permettent de telles expériences, notamment lorsque les stagiaires sont exposés à différents contextes de pratique, soit au fait d être différents, ou aux occasions d'apprécier des stratégies locales utilisées pour des phénomènes plus globaux (adoption, trafic humain, immigration, etc.). Ces expériences comprennent également l'exposition des stagiaires à certaines valeurs locales, comme dans un contexte thailandais celle qui consiste ne pas perdre la face, ou encore, à son fatalisme, voire à l'inévitabilité du destin (Engstrom et Jones, 2007). Pour leur part, Rankopo et Osei-Hwedie (2011) discutent à propos de la nécessité de se libérer d'une vision eurocentrique ou américanocentrique en travail social afin d'adopter des pratiques qui soient culturellement appropriées en fonction de la diversité des contextes locaux que les stagiaires investissent.

Das et Carter Anand (2012) soulèvent davantage la nécessité de réfléchir de façon critique dans un contexte international et présentent des outils à cet effet, soit le travail narratif, le debriefing, le questionnement réflexif visant la déconstruction des pratiques et l'étude d'incidents critiques. Ces outils permettent de remettre en question les croyances personnelles et professionnelles et 
"Les stagiaires doivent être en mesure de poser un regard critique sur les valeurs, les principes et les normes de leur pays d'origine. Il devient ainsi nécessaire de créer davantage d'espaces permettant de se questionner non seulement durant le stage, mais aussi avant et après celui-ci. » également de réinterpréter la réalité. Pour favoriser ce processus réflexif, Das et Carter Anand (2012) suggèrent un partenariat entre l'étudiante ou étudiant et l'éducatrice ou éducateur qui fournit «sécurité émotionnelle, confiance et reconnaissance des besoins des étudiantes et des étudiants " (p. 10 [notre traduction]). De son côté, tout en reconnaissant les mérites des stages internationaux, Razack (2002) soulève également leurs dangers. Bien qu'ils permettent d'enrichir le curriculum de formation, ces stages peuvent aussi constituer une activité colonisante. Les stagiaires doivent être en mesure de poser un regard critique sur les valeurs, les principes et les normes de leur pays d'origine. Il devient ainsi nécessaire de créer davantage d'espaces permettant de se questionner non seulement durant le stage, mais aussi avant et après celui-ci.

Offrir des stages internationaux suppose une nécessaire préparation des stagiaires au moment de s'engager dans pareille expérience. Bell et Anscombe (2013) abordent la préparation au départ et insistent sur l'importance de lire à propos du travail social international et de réfléchir aux valeurs professionnelles afin d'avoir une meilleure compréhension en matière de droits de la personne et de justice sociale. Pour leur part, Nuttman-Shwartz et Berger (2012, p. 238 [notre traduction]) indiquent qu' " un aspect essentiel de la préparation porte sur l'enseignement des dimensions philosophique, éthique et pratique du travail social international ». Par exemple, la préparation à un stage en sol africain doit s'inspirer de leçons d'organismes de développement qui permettent d'identifier des dimensions nécessaires à la bonne connaissance du contexte historique (colonisation, mondialisation, dette nationale, etc.) et des enjeux éthiques liés à la sécurité personnelle (risque d'exposition à la criminalité) ou à la gestion du risque (santé et VIH/SIDA) (Heron, 2005). La préparation aux stages internationaux sous-tend aussi la reconnaissance de l'impact des identités et des expériences personnelles sur les apprentissages professionnels; en ce sens, des stagiaires LGBT ou ayant un handicap pourraient vivre des situations difficiles en raison de certaines valeurs du pays d'accueil (Mathiesen et Lager, 2007). Enfin, la préparation des stagiaires nécessite d'explorer les 
aspects cognitifs (relations de pouvoir entre le pays d'origine et le pays d'accueil), émotionnels (façon d'être perçu ou traité dans le pays d'accueil) et culturels (comportements différents dans la communauté d'accueil) (Nuttman-Shwartz et Berger, 2012).

Epprecht (2004) mentionne de nombreux exemples où les personnes en situation d immersion sont confrontées à des dilemmes éthiques issus d'attentes irréalistes du milieu de stage à l'égard de l'aide qu'elles peuvent apporter, d'observations de pratiques non éthiques, de lois portant atteinte à leur sécurité ou de comportements qui discriminent certaines d'entre elles. Non seulement ces personnes séjournant à l'étranger sont « désorientées sur le plan de leurs propres croyances de ce qui est bien ou mal ", mais elles se retrouvent parfois dans des contextes où «leur capacité de prendre des décisions éthiques est compromise " (Epprecht, 2004, p. 692; p.695 [notre traduction]). Ces diverses perspectives soulevées dans la littérature confirment l'importance d'outiller les éventuels stagiaires à l'international en leur permettant d'intégrer les notions associées à l'analyse intersectionnelle et à la résolution de dilemmes éthiques.

\section{L'analyse intersectionnelle pour réfléchir aux enjeux éthiques dans un contexte de stage international}

À la lumière des enjeux éthiques que rencontrent les étudiantes et les étudiants qui choisissent comme expérience pratique en travail social un stage à l'international, l'intersectionnalité se veut un cadre d'analyse pertinent pour comprendre la complexité des situations rencontrées. L'analyse intersectionnelle permet d'explorer des phénomènes complexes en tenant compte des structures de domination en place (Hulko, 2009) afin de combattre les inégalités dans une perspective de justice sociale (Lanteigne et Savoie, 2010). Ce courant d'analyse est issu de la critique de femmes afro-américaines qui disaient vivre de l'exclusion dans les théories féministes existantes, que ces dernières occultaient leur vécu, rendaient leur quotidien invisible et elles, sans voix (Bilge, 2010; Hulko, 2009). Ces femmes noires critiquaient la 
"À la lumière des enjeux éthiques que rencontrent les étudiantes et les étudiants qui choisissent comme expérience pratique en travail social un stage à l'international, l'intersectionnalité se veut un cadre $d$ 'analyse pertinent pour comprendre la complexité des situations rencontrées."

"L'analyse intersectionnelle suppose " de prendre en compte la diversité des statuts sociaux et des expériences des femmes, tout comme la pluralité des composantes identitaires qui en découlent " (Marchand et Ricci, 2010, p. 65)." vision hégémonique du féminisme de la deuxième vague (Bilge, 2009) et remettaient en question la nature essentialiste de la catégorie "genre ", voyant cette affirmation de l'identité comme étant plus restrictive et régulatrice qu'émancipatrice (Lanteigne et Savoie, 2010). Elles revendiquaient une politique qui s'engage dans « la lutte contre l'oppression raciste, sexuelle, hétérosexuelle et de classe " (Combahee River Collective, 2008). Composée de femmes noires de Boston, la collective Combahee River s'était donné comme but de "développer une analyse et une pratique basée sur le fait que les principaux systèmes d'oppression sont imbriqués "(Combahee River Collective, 2008, p. 60). Le concept d'intersectionnalité, plus précisément, est attribué à deux théoriciennes féministes noires, Kimberle Crenshaw, première à utiliser ce concept, et Patricia Hill Collins, théoricienne du Black Feminism (Bilge, 2009). Dans un texte fondamental de la pensée intersectionnelle écrit en 1991 et traduit en 2005, Crenshaw met en perspective, dans le cas de violence contre les femmes de couleur, comment elles sont marginalisées tant parce qu'elles sont femmes que parce qu'elles sont noires. L'analyse intersectionnelle suppose " de prendre en compte la diversité des statuts sociaux et des expériences des femmes, tout comme la pluralité des composantes identitaires qui en découlent " (Marchand et Ricci, 2010, p. 65). C'est dans ce sens que les auteures Bilge (2009) et Hulko (2009) parlent d'identités multiples (femme, lesbienne, noire, pauvre, etc.) qui s'entrecroisent avec des systèmes d'oppression ou ce que Hill Collins (1990) nomme "la matrice de la domination " (patriarcat, sexisme, racisme, etc.).

Shields (2008) explique qu'il existe plusieurs conceptualisations de l'intersectionnalité selon les perspectives théoriques qui y sont rattachées. Cependant, un point reste commun pour comprendre ce cadre d'analyse, soit le fait que les catégories qui définissent l'identité sociale et qui construisent les relations sociales se coconstituent, c'est-à-dire qu'une catégorie, comme le genre, prend son sens si elle est en relation avec d'autres catégories, comme la race ou la classe sociale. L'auteure ajoute également que l'analyse intersectionnelle met en lumière les réalités de la vie et qu'il n'y a pas de catégorie identitaire unique. L'identité n'est pas fixe 
"Il importe de saisir comment les oppressions et les privilèges particuliers que vivent des individus selon leur contexte géographique, historique et culturel sont mis en relation avec des identités multiples et permettent l'analyse des situations d'inégalité sociale. " et peut changer dans le temps et l'espace, ce qui signifie que les catégories peuvent se transformer en fonction du contexte historique et culturel.

Crenshaw (2005) distingue deux niveaux d'analyse intersectionnelle, le niveau structurel et le niveau politique. Se référant à Crenshaw, Shields (2008) mentionne que l'intersectionnalité structurelle renvoie à la façon dont le statut légal ou les besoins sociaux marginalisent les personnes à cause surtout des diverses facettes de leurs identités. En fait, Crenshaw (2005) explique qu'une analyse intersectionnelle structurelle lui a permis de constater que les femmes noires occupent des positions sociale, économique et politique différentes de celles des femmes blanches. Selon elle, trop de femmes de couleur sont non seulement aux prises avec des expériences quotidiennes de sexisme et de racisme, mais aussi de pauvreté, de sous employabilité et de chômage.Ainsi, les structures sociales et les systèmes d'aide en place répondent peu aux besoins particuliers de ces femmes qui vivent des expériences d'exclusion. Dans un autre ordre d'idées, Crenshaw (2005) soulève qu'une analyse intersectionnelle politique permet la mise en lumière des besoins et des buts différents de certains groupes et le potentiel conflictuel de leurs revendications respectives. Une analyse intersectionnelle politique suggère ainsi d'examiner la manière dont les discours antiracistes et féministes pris séparément ne parviennent pas à rendre compte des sources d'oppression et arrivent difficilement à comprendre les imbrications du genre et de la race. Comme l'explique Crenshaw, les discours féministes et antiracistes contemporains ont échoué dans leur tentative de comprendre les identités des femmes racisées. C'est dans ce sens que hooks $(2008$, p. 115) dira que "pour qu'un nouvel ordre social émerge, il faut contester et changer la politique de domination telle qu'elle se manifeste dans l'oppression impérialiste, capitaliste, raciste et sexiste ». Enfin, il s'agit de mieux saisir en quoi les interrelations des différentes expressions des identités sociales entrent en intersection avec des systèmes de domination constituant des expériences singulières d'oppression et de privilège. Il importe de saisir comment les oppressions et les privilèges particuliers que vivent des individus selon leur contexte 
géographique, historique et culturel sont mis en relation avec des identités multiples et permettent l'analyse des situations d'inégalité sociale. À cet effet, Bilge (2009, p. 73) est claire :

Pour nombre d'auteurs, l'intersectionnalité doit constituer un cadre d'analyse permettant d'aborder des questions aussi bien macrosociologiques que microsociologiques. L'analyse intersectionnelle opère à deux niveaux. Au niveau microsocial, par sa considération des catégories sociales imbriquées et des sources multiples de pouvoir et de privilège, elle permet de cerner les effets des structures d'inégalités sur les vies individuelles et les manières dont ces croisements produisent des configurations uniques. Au niveau macrosocial, elle interroge les manières dont les systèmes de pouvoir sont imbriqués dans la production, l'organisation et le maintien des inégalités.

Bilge (2010, p. 59) réclame une perspective intersectionnelle holiste selon laquelle "les différents éléments constituant le système sont liés autant par leurs similitudes que leurs différences » et qu'elle propose en opposition à la perspective moniste ou pluraliste. Weldon (2006) et Bilge (2010) indiquent que le modèle additif et le modèle multiplicateur réduisent l'analyse à de simples formules mathématiques et ne rendent pas compte de la complexité intersectionnelle des différents axes de domination et de privilège. C'est pour ces raisons, entre autres, que Bilge (2010) privilégie la perspective holiste pour cerner les axes de domination. Par ailleurs, Crenshaw (2005) mentionne qu'il importe de remettre en question la valeur attribuée aux catégories de façon à ne pas présumer que l'une est plus importante que l'autre. Comme elle l'explique (p. 76), « le problème le plus pressant rencontré dans la plupart des cas, sinon dans tous, n'est pas l'existence de catégories, mais bien les valeurs particulières qui leur sont attachées, et la manière dont ces valeurs créent les hiérarchies sociales et les entretiennent ». C'est ainsi que comprendre la valeur des catégories part du narratif, c'est-à-dire, de la façon dont les histoires et les 
"Dans un contexte international, les stagiaires doivent se soucier de faire émerger le narratif des personnes accompagnées pour mieux saisir le sens de leur vécu.»

"Les stagiaires arrivent avec un bagage culturel qui est souvent confronté par ce nouvel environnement; leurs repères normatifs et professionnels sont parfois ébranlés, les plaçant devant des situations de malaise. " trajectoires des personnes vont privilégier des dimensions de l'expérience plutôt que d'autres. En ce sens, il s'agit de saisir quelles dimensions de l'expérience sont significatives. Dans un contexte international, les stagiaires doivent se soucier de faire émerger le narratif des personnes accompagnées pour mieux saisir le sens de leur vécu.

Dans le cadre d'un stage international, il importe aussi de se pencher sur les axes de domination et de privilège qui different d'un contexte national et culturel à un autre (Weldon, 2006). Les stagiaires qui se retrouveraient par exemple dans un contexte arabo-musulman devraient tenir compte des paramètres susceptibles de diverger de ceux retrouvés dans un contexte européen, et ce, d'autant plus s'ils interviennent dans un contexte européen auprès d'une femme immigrante ou réfugiée. Les axes de domination et de privilège varieront pour la femme musulmane selon sa situation géographique, si elle est dans son pays d'origine ou si elle est immigrante. Il reste que nonobstant le lieu où cette femme se retrouve, les catégories "femme ", " algérienne " et "musulmane ", par exemple, demeurent; mais elles doivent être analysées à partir des systèmes d'oppression et de privilège en place. Qui plus est, ces systèmes doivent être compris en tenant compte de la trajectoire de vie de la personne et de la situation sociale, économique et politique, de façon à éclairer les rapports qui se construisent entre les différents axes d'oppression et de privilège. Selon hooks (2008, p. 128), « les comportements n'ont pas le même sens selon le contexte culturel dans lequel ils s'inscrivent : ce qui est acceptable pour telle culture ne le sera pas par ailleurs ». Il s'agit donc d'apprendre à décrypter les codes culturels qui se présentent en stage. Il ne faut pas craindre la différence, mais la comprendre pour mieux l'apprécier. Cette différence exprimée tant dans les codes culturels, les valeurs, les comportements et les modes de communication doit être dévoilée pour permettre la communication interculturelle. Enfin, dans l'analyse intersectionnelle, les étudiantes et étudiants canadiens, selon leurs identités respectives, doivent également procéder à l'analyse de leurs privilèges, puisqu'ils proviennent d'un pays dit démocratique, fondé sur la liberté individuelle et la sécurité — à 
plus forte raison, s'ils sont blancs et de classe moyenne. Les stagiaires arrivent avec un bagage culturel qui est souvent confronté par ce nouvel environnement; leurs repères normatifs et professionnels sont parfois ébranlés, les plaçant devant des situations de malaise. En ce sens, Epprecht (2004) soutient qu'il arrive que le nouveau contexte désoriente et affecte la perception de ce qui est bien et de ce qui est mal. Ces situations exposent ainsi les stagiaires à des dilemmes éthiques avec lesquels ils doivent apprendre à composer.

\section{La résolution de dilemmes éthiques dans des contextes qui déstabilisent}

"Un dilemme éthique se présente quand une personne fait face à une décision à prendre entre des scénarios possibles et contradictoires. "
Un dilemme éthique se présente quand une personne fait face à une décision à prendre entre des scénarios possibles et contradictoires. Il se caractérise par la nécessité de devoir choisir, voire décider, dans le contexte d'une situation où valeurs, responsabilités, devoirs ou obligations se confrontent et font appel à des choix différents qui entraînent « des conséquences à la fois positives et négatives sur soi, sur autrui ou sur l'environnement " (Legault, 1999, p. 107). Selon Albert (2006, p. 77), la résolution d'un dilemme éthique invite à une démarche de

réflexion sur l'action qui suppose une conscience de soi et des enjeux éthiques en présence dans une situation donnée. Cette démarche veut éclairer une décision professionnelle et suppose, entre autres, une réflexion sur les valeurs confrontées par la décision (au nom de quoi) et une conscience des conséquences sur les personnes touchées par la décision (effets de la décision). Ce processus comprend un certain nombre de stratégies mises en œuvre par l'intervenante pour l'accompagner dans sa démarche réflexive.

Des auteurs comme Legault (1999), Mattison (2000) et Reamer (1995) se sont penchés sur la résolution des dilemmes éthiques en 
«Ce commentaire rappelle l'importance du questionnement des travailleuses ou travailleurs sociaux dans leur pratique quotidienne et la nécessité d'établir un dialogue, c'està-dire, une réflexion dialogique avec l'Autre, pour permettre une recherche visant la meilleure conduite à tenir.» proposant des modèles, voire des grilles, qui systématisent les étapes menant à une décision. Une première dimension que partagent ces trois modèles et qui est incontournable dans la résolution de dilemmes éthiques consiste en la prise en compte des valeurs en conflit. Une autre dimension commune à ces trois modèles est la référence au code de déontologie, bien que dans celui de Legault elle ne soit pas explicitement nommée. Une troisième consiste, vers la fin de la démarche, à justifier ou à expliciter le positionnement ou la décision. Enfin, ces auteurs s'entendent sur l'importance accordée à l'exploration des scénarios. Toutefois, il est clair pour eux que la dimension processuelle, souvent itérative, de la démarche réflexive vers une décision est valorisée et essentielle à la prise de décision éthique. Cependant, Reamer (1995) met en garde sur le fait que ce processus n'assure pas des résultats clairs et sans équivoque. En effet, de par leur nature, les dilemmes éthiques revêtent souvent une grande complexité. Ainsi, Reamer (1995) explique que devant des situations qui renverraient à des enjeux éthiques similaires les analyses de diverses intervenantes peuvent fort bien produire des résultats différents. Il précise (p. 80 [notre traduction]) : «Cela est bien, particulièrement si nous convenons que le dialogue continu entre praticiennes ou patriciens en regard des perspectives propres à chacun enrichira potentiellement leur compréhension et leur réflexivité. Comme dans toute autre dimension du travail social, c'est le processus qui compte le plus.» Ce commentaire rappelle l'importance du questionnement des travailleuses ou travailleurs sociaux dans leur pratique quotidienne et la nécessité d'établir un dialogue, c'est-à-dire, une réflexion dialogique avec l'Autre, pour permettre une recherche visant la meilleure conduite à tenir.

Au-delà du processus de délibération éthique auquel renvoient ces modèles, des auteurs (Legault, 1999; Reamer, 1995; Mattison, 2000) insistent sur le raisonnement qui doit guider la réflexion. Legault (1999), pour sa part, identifie deux approches pour accompagner le processus décisionnel et justifier le rationnel de la décision. L'une d'elles est qualifiée de déontologique et renvoie à un argumentaire de nature prescriptive, certaines valeurs ou normes devant être respectées. L'autre approche est qualifiée de 
conséquentialiste (téléologique) et renvoie à un argumentaire basé sur les conséquences des options décisionnelles envisagées (Albert, 2006). Reamer (1995) réfere aussi à ces formes d'argumentaire qui s'inspirent de théories éthiques. Par ailleurs, il insiste sur l'attention qui devrait être accordée à l'évaluation des conséquences de la décision. À cet effet, il précise que peu importe l'approche privilégiée, il demeure essentiel de se préoccuper des conséquences des décisions éthiques. De son côté, Mattison (2000, p. 203 [notre traduction]) souligne : «Bien que les travailleuses et travailleurs sociaux, dans leur processus de décision éthique, ne s'expriment pas habituellement en des termes philosophiques, leur réflexion est, d'une manière consciente ou non, empreinte d'éléments déontologiques et téléologiques.»

L'étude de diverses grilles de résolution de dilemmes éthiques permet d'apprécier les dimensions particulières sur lesquelles insistent les auteurs respectifs. Legault (1999) fait falloir l'importance de tenir compte des émotions dominantes, Quintin et Boire-Lavigne (2013) notent la nécessité de la prise en compte du vécu généré par la situation et du sens qu'elle revêt pour les personnes impliquées et Mattison (2000) met en lumière la pertinence de considérer les facteurs personnels dans la prise de décision éthique, notamment la conscience de soi, de son bagage de valeurs et de son système de préférence. De plus, Reamer (1995) recommande de consulter des collègues et des experts dans un esprit de dialogue. Pour Legault (1999), cette consultation suppose "dans une culture démocratique axée sur les droits de la personne, de dépasser le "je" pour construire un

"Si les questions éthiques se présentent de façon quotidienne dans la pratique $d u$ travail social, elles le sont tout autant, sinon davantage, dans un contexte de stage international. » "nous" enraciné dans un contexte historique et social " (p. 191). Il propose ainsi de procéder à une réflexion critique et dialogique sur le "caractère universalisable des raisons d'agir ", à partir de trois critères, notamment, l'impartialité, la réciprocité et l'exemplarité (p. 277). L'analyse de la décision en fonction de ces critères permet de faire le point sur la décision et de prendre un recul nécessaire à son évaluation.

Si les questions éthiques se présentent de façon quotidienne dans la pratique du travail social, elles le sont tout autant, sinon davantage, dans un contexte de stage international. Selon Sewpaul 
(2003), ce type d'expérience déstabilise les stagiaires et leur crée des occasions de remettre leurs connaissances en question. Pour la personne qui doit décider de la meilleure conduite à tenir, plus la situation est perçue comme étant lourde de conséquences, plus le sentiment de déchirement est marqué. Dans un contexte international, les stagiaires peuvent avoir l'impression de perdre leurs repères en certaines occasions (Epprecht, 2004). Plus l'univers dans lequel baignent ces étudiantes ou étudiants est différent de celui laissé derrière, voire de leur milieu habituel de vie, plus ce nouvel environnement risque de les confronter à des situations nouvelles au sein desquelles leurs valeurs, celles qui sont déjà intégrées, sont ébranlées.

\section{Exemples de situations qui génèrent des dilemmes éthiques}

Lors de stages internationaux, plusieurs situations complexes se présentent à la praticienne ou au praticien en devenir. L'expérience des vingt-cinq dernières années à l'ÉTS de l'Université de Moncton a permis de constater différents dilemmes éthiques rapportés par les stagiaires pendant leur séjour à l'étranger ou à leur retour, notamment autour des trois situations suivantes : des épisodes de violence dans leur quotidien, par exemple, dans la rue à l'égard de personnes immigrantes ou itinérantes; des propos homophobes exprimés dans leur milieu de stage, leur famille d'accueil ou leur nouveau réseau social; et des interventions qui incluent la punition corporelle comme moyen de discipline pour des jeunes en contexte institutionnel. Examinons plus en détail la façon dont pourraient se dérouler ces trois situations.

Dans la première, imaginons une stagiaire canadienne qui marche dans la rue. Elle est accompagnée d'un collègue du pays d'accueil. Les deux sont témoins d'une agression envers un itinérant. La stagiaire réagit avec surprise et indignation. Elle interpelle son collègue dans l'intention d'intervenir. Ce dernier déclare que ce type d'agression est monnaie courante et il incite la stagiaire à ne pas s'en mêler. Celle-ci est donc déchirée, à un 
"...une stagiaire

se retrouve dans un

milieu de l'enfance

où les méthodes

disciplinaires

impliquent une

forme de punition

corporelle qui contraste

essentiellement avec ce

qu'elle a convenu selon

ses valeurs comme

étant approprié. Or,

comme c'est la façon

de faire du milieu, culturellement acceptée

dans ce contexte, comment arrivera-t-

elle à gérer ce genre de situation qui heurte profondément ses valeurs?" premier niveau, entre intervenir ou non - entre la protection de l'autre et sa propre sécurité. À un second niveau, elle est tiraillée entre le désir d'intervenir pour protéger l'itinérant — par exemple en faisant appel aux forces policières - et le respect de ce que son collègue considère comme normal dans les circonstances, dans le respect des pratiques locales, évitant ainsi d'évaluer la dangerosité de la situation en fonction de sa propre perspective. Pour Beck (2003), un environnement nouveau, qu'il soit similaire ou non à celui de son pays d'origine, provoque une réflexion sur la façon dont les personnes doivent être traitées. De plus, Dubois et Ntetu (2000) ajoutent que lors de contacts interculturels à l'international, les stagiaires ne comprennent pas toujours ce qui est attendu d'eux dans certaines situations.

La deuxième situation concerne l'exposition d'un stagiaire à des propos homophobes, celui-ci étant particulièrement interpellé par les enjeux de justice sociale sous-jacents à cette question. Une professionnelle qui l'encadre lors de son stage tient fréquemment en sa présence un discours infériorisant à l'égard des personnes homosexuelles. Le stagiaire se retrouve déchiré entre son désir de promouvoir la justice sociale en dénonçant les propos homophobes et son besoin de maintenir la relation et de respecter les valeurs personnelles, culturelles ou religieuses sur lesquelles peut reposer ce discours. On peut alors imaginer l'ampleur du malaise de stagiaires LGBT quand cette dimension de leur identité ne peut être dévoilée ni partagée dans des contextes qui risquent de les ostraciser. Ce genre de situation qui renvoie à des composantes identitaires fondamentales est difficile à vivre (Mathiesen et Lager, 2007).

Dans la troisième situation, une stagiaire se retrouve dans un milieu de l'enfance où les méthodes disciplinaires impliquent une forme de punition corporelle qui contraste essentiellement avec ce qu'elle a convenu selon ses valeurs comme étant approprié. Or, comme c'est la façon de faire du milieu, culturellement acceptée dans ce contexte, comment arrivera-t-elle à gérer ce genre de situation qui heurte profondément ses valeurs? Elle pourrait avoir envie d'agir, de se porter à la défense de l'enfant avec toutes les conséquences que cela peut comporter, un geste 
"...il est de la

responsabilité

de l'ÉTS de

bien préparer les stagiaires avant leur départ et de bien encadrer à distance leurs expériences d'apprentissage de façon à leur permettre d'intervenir avec les outils appropriés. " qui pourrait aussi être perçu comme étant teinté d'une attitude colonialiste en imposant des valeurs à un pays d'accueil. Or, choisir de ne pas agir peut produire chez la stagiaire un sentiment d'échec, ou d'ambiguiité face d'une part à son désir de réussir sa formation à l'international et d'autre part face à la protection de l'enfant qui subit dans ce contexte une forme de violence qui serait proscrite dans un pays comme le nôtre, et qui va aussi à l'encontre de l'article 19 de la Convention relative aux droits de l'enfant (Organisation des Nations unies, 1989).

Il est donc fondamental, comme formatrices ou formateurs en travail social et responsables de stages internationaux qui incluent de telles expériences, d'en être conscients et de se soucier de la capacité des stagiaires à gérer de tels dilemmes. Plus encore, il est de la responsabilité de l'ÉTS de bien préparer les stagiaires avant leur départ et de bien encadrer à distance leurs expériences d'apprentissage de façon à leur permettre d'intervenir avec les outils appropriés. Cet encadrement devrait donner l'occasion de réaliser un processus de réflexion éthique et critique sur la meilleure conduite à tenir (Lamoureux, 2003).

Ces trois situations sont aussi pertinentes l'une que l'autre et se prêteraient également à un exercice de délibération éthique à partir d'une perspective intersectionnelle. Cependant, pour illustrer notre modèle d'analyse, nous nous en tiendrons à la troisième, étant donné sa prévalence dans les expériences vécues par les stagiaires de l'ÉTS et la profondeur du malaise qu'elle suscite. Pour démontrer le processus de réflexion et de décision éthiques dans un contexte de stage international, nous proposons le modèle suivant qui sera appliqué à cet exemple fictif, bien qu'inspiré de situations qui se sont présentées à des stagiaires.Alors que les étapes du processus proposé se réalisent normalement et essentiellement de façon itérative, nous proposons aux fins de l'exercice de les traiter de manière séquentielle, à partir du centre, puis vers le cercle supérieur central pour aller ensuite vers la droite. Il est à noter que les principes de l'intersectionnalité qui sont au cœur de ce modèle traversent chacune des étapes.Aussi, ces dernières sont inspirées des modèles de délibération proposés par Quintin et Boire-Lavigne (2013), Legault (1999) et Mattison (2000). 
Figure 1 : Modèle de délibération éthique intersectionnelle.

La réflexion critique et dialogique sur le « caractère universalisable des raisons d'agir » (Legault, 1999, p. 277), soit l'impartialité, la réciprocité et l'exemplarité.
Les personnes impliquées,

moi, les autres et

l'environnement

\section{La situation, le dilemme et}

l'option spontanée

ET

L'intersectionnalité
Les émotions, le vécu et le sens généré par la situation
La décision et son évaluation (raisonnement conséquentialiste ou déontologique)
Les conséquences positives et négatives, sur soi, sur les autres et sur l'environnement
Les valeurs personnelles, professionnelles et sociales
Les normes professionnelles, sociales, juridiques et culturelles 


\section{La situation, le dilemme et l'option spontanée}

En reprenant le dilemme que pose le fait d'être témoin d'une punition corporelle, imaginons la situation suivante. Dans un centre d'accueil pour jeunes en difficulté, deux garçons de cinq ou six ans s'adonnent à des attouchements impudiques. Surpris sur le fait par un employé, les jeunes sont aussitôt conduits à la direction de l'établissement. La conséquence imposée consiste en l'administration de dix fessées en guise de dissuasion d'un tel acte associé à l'homosexualité, jugée répréhensible par certains employés. La stagiaire est présente au moment de la correction; elle ne comprend pas tout à fait le contexte et elle éprouve un malaise face à la situation. Échangeant par la suite avec une intervenante du centre, elle cherche à comprendre ce qui se produit. L'intervenante lui explique les raisons d'une telle punition, ajoutant que cette pratique courante vise à décourager tout comportement déviant. Le dilemme qui se pose ici pour la stagiaire consiste à savoir si elle doit intervenir auprès de la direction et des employés pour les sensibiliser à d'autres formes de discipline ou si elle doit se taire et ne pas s'impliquer outre mesure. La stagiaire choisit de garder le silence.Avant d'explorer les dimensions importantes de chaque étape de la délibération éthique, il s'agit d'effectuer un premier niveau d'analyse intersectionnelle de la situation.

\section{L'intersectionnalité}

Dans une analyse intersectionnelle appliquée à la résolution de dilemme éthique, la stagiaire confrontée à un tel dilemme doit s'interroger sur les quatre dimensions de cette analyse (systèmes de privilèges et d'oppression, identités, contextes et expériences vécues). D'abord, elle doit réfléchir sur sa propre position d'étrangère et aux privilèges que cette position lui confere (Marchand et Ricci, 2010). Par exemple, le fait d'être nord-américaine peut lui attribuer un statut privilégié; elle pourrait être perçue comme ayant accès à des ressources abondantes et jouissant d'une vaste 
"Dans une analyse intersectionnelle appliquée à la résolution de dilemme éthique, la stagiaire confrontée à un tel dilemme doit s'interroger sur les quatre dimensions de cette analyse (systèmes de privilèges et d'oppression, identités, contextes et expériences vécues). "

"Par exemple, les stagiaires doivent prendre conscience de leurs prétentions à l'égard des manières de faire de l'Autre. Avoir pour réflexe de disqualifier les formes d'intervention supposant qu'on sait mieux faire cheznous, qu'on est plus évolué." expertise professionnelle. Elle doit aussi prendre en compte la position privilégiée qu'occupe le directeur de l'institution qui jouit d'une reconnaissance sociale dans son milieu et qui aura, entre autres, à témoigner de la réussite ou de l'échec du stage. Qui plus est, Epprecht (2004) ajoute que la possibilité de ne pas obtenir une lettre de recommandation ou que des amis du pays d'accueil subissent des répercussions négatives influence la prise de décision éthique en contexte de stage international. Par ailleurs, les employés de l'institution ne sont pas nécessairement tous d'accord avec la pratique de la fessée. Or, ceux qui seraient en désaccord pourraient ne pas bénéficier du privilège d'exprimer leur point de vue par crainte de conséquences. Il faut souligner que dans cette situation, les deux enfants - et, par extension, leurs camarades du centre - ne jouissent pas de privilège en raison de leur âge et de leur statut.

À l'égard de la prise en compte des systèmes d'oppression, les stagiaires doivent réfléchir à ceux qui sont en présence comment ils s'articulent entre eux et comment ils se renforcent ou s'éliminent (Bilge, 2009) — et s'interroger sur des systèmes d'oppression qui sont en jeu dans la situation (colonialisme, sexisme, âgisme, etc.). Par exemple, les stagiaires doivent prendre conscience de leurs prétentions à l'égard des manières de faire de l'Autre. Avoir pour réflexe de disqualifier les formes d'intervention supposant qu'on sait mieux faire chez-nous, qu'on est plus évolué. En ce sens, Razack (2002) explique que des étudiantes ou des étudiants amorcent le stage avec une attitude teintée de supériorité, de tristesse, voire de pitié, devant des personnes marginalisées, exclues ou discriminées dans le pays hôte. Ainsi, dans l'exemple de la fessée imposée comme punition aux enfants pour les gestes impudiques posés, et sur la base des différences observées, la stagiaire doit se questionner sur son interprétation et éviter de généraliser en attribuant une telle pratique à l'ensemble d'une culture essentiellement différente de la sienne.

Sur le plan des identités, les stagiaires doivent aussi réfléchir à savoir en quoi la question du genre est significative, ou non, pour chacune des parties. Dans notre exemple, le fait que ce sont des 
garçons plutôt que des filles est-il en cause? En ce qui a trait à l'âge, il s'agit de se pencher sur le statut qui lui est accordé dans le pays d'accueil. L'âge des enfants est-il ici en cause, bien que la communauté internationale reconnaisse aux enfants de tous les âges le statut d'êtres de droit à part entière (Organisation des Nations unies, 1989)? De plus, le statut social et la race de l'enfant ont-ils une influence sur la conception que s'en font les personnes concernées par rapport à l'évènement, c'est-à-dire, l'enfant luimême, l'adulte qui corrige, et des employés et la stagiaire qui en sont témoins? Cette dernière doit enfin s'interroger quant à la reconnaissance hiérarchique accordée au statut professionnel pour toutes les personnes impliquées dans la situation.

Il importe d'analyser le contexte dans lequel la situation se produit. Selon le pays d'accueil, des stagiaires de l'ÉTS rapportent à l'occasion qu'en fonction du climat social, politique ou économique ambiant, des insécurités peuvent surgir et avoir un impact sur eux, sur les individus et sur la communauté, tant dans les comportements que dans les attitudes. Ici, est-ce que le contexte institutionnel, culturel ou religieux influence l'interprétation de l'utilisation de la punition corporelle comme pratique disciplinaire? Le contexte (familial, rural, minoritaire, ou autre) dans lequel la stagiaire a évolué peut-il avoir une incidence sur l'analyse qu'elle aura de la situation? En fonction de ses expériences personnelles, quel serait l'apport d'avoir ou non vécu, ou d'avoir ou non été témoin, d'actes de violence physique sur l'appréciation qu'elle se fait de l'évènement? En quoi ses expériences et celles des employés colorent-elles leur capacité d'affirmer leurs positions respectives face à ce moyen de correction? De plus, si la stagiaire croit que l'enfant doit être respecté dans son intégrité et avoir le droit de s'exprimer et d'être protégé, ne risque-t-elle pas d'évaluer la situation à la lumière de ses convictions personnelles? 


\section{Les personnes impliquées, moi, les autres et l'environnement}

À cette étape, il est nécessaire d'explorer à travers les narratifs des acteurs, en quoi le contexte, les identités des personnes impliquées et les expériences de privilège et d'oppression vécues s'articulent pour guider l'analyse (Crenshaw, 2005). En présence d'un dilemme éthique, les stagiaires doivent tenir compte de l'ensemble des personnes impliquées dans la situation. Dans la situation qui nous concerne, outre les personnes déjà nommées, soit les enfants, les intervenants, la direction et la stagiaire, il faudrait, par exemple, se soucier des autres enfants dans l'institution, les partenaires communautaires ou la famille élargie, si elle est toujours présente dans la vie de chacun des deux enfants. Aussi, la communauté environnante pourrait selon la perméabilité de l'institution être interpellée par cette situation. Par ailleurs, si l'institution est subventionnée par un organisme philanthropique, gouvernemental ou non gouvernemental, il faut le considérer, tel que l'analyse intersectionnelle politique le propose, question de tenir compte du potentiel conflictuel des revendications de chacun des groupes en présence.

\section{Les émotions, le vécu et le sens générés par la situation}

Un dilemme éthique ne se pose pas sans générer des émotions (Legault, 1999). En fait, ce sont elles qui soulèvent chez les stagiaires un malaise et le sentiment de se retrouver face à un dilemme éthique, devant lequel il est nécessaire d'explorer ses émotions, les éléments de son vécu qui se rattachent à la situation et du sens à donner à cette situation, notamment en fonction de ses identités. Cette conscience de son histoire personnelle permet d'apprécier la valeur accordée à une catégorie par rapport à une autre (Crenshaw, 2005). Il faut aussi se préoccuper des émotions, du 
vécu et du sens que la situation génère pour chacune des personnes impliquées, en fonction de leurs identités. De plus, Barlow (2007) explique que la rencontre de deux mondes (pays d'origine et pays hôte) constitue parfois un moment intense sur le plan émotionnel pouvant se traduire par le jugement, la condamnation ou le désespoir. Par contre, cela peut aussi déclencher un sentiment de pitié qui embrouille le regard sur la situation.

\section{Les normes professionnelles, sociales, juridiques et culturelles}

Après avoir exploré le contexte d'origine des stagiaires et son impact sur les émotions ressenties, il importe de prendre en considération le contexte axiologique et normatif du pays d'accueil. En fait, tout ce qui est normatif est par définition prescriptif. Ainsi, la conduite à tenir devant la situation présentée est définie par diverses structures normatives ou législatives. Les stagiaires devront tenir compte des normes professionnelles acquises en vertu du code de déontologie en vigueur dans leur contexte de formation et de pratique au Canada et connaitre le cas échéant les normes professionnelles de leur pays d'accueil. Les stagiaires auront à s'informer des diverses lois et conventions qui régissent la pratique, dans le champ de l'enfance et des institutions impliquées, notamment la Convention relative aux droits de l'enfant (Organisation des Nations unies, 1989) et connaitre les conduites et les comportements qui sont conformes aux codes sociaux et culturels de leur pays d'accueil. Certains codes ou lois peuvent à l'occasion contribuer à générer ou à maintenir des systèmes d'oppression dont une ou plusieurs personnes impliquées peuvent faire l'objet. Epprecht (2004, p. 696 [notre traduction]) propose l'exemple suivant :

Une loi sud-africaine exige que les personnes qui connaissent l'existence d'une situation de violence domestique ou de maltraitance d'enfant en fassent un signalement aux forces policières. 
Cependant, en l'absence de recours ou de moyens pour assurer un suivi au signalement (refuges, counseling, force policière sensible à la question du genre), un tel signalement peut contribuer à ce que la victime subisse d'autres violences. Comment, à cet égard, évaluer s'il est préférable sur le plan éthique de respecter la loi ou d'y contrevenir?

Ainsi, l'intersectionnalité structurelle proposée par Crenshaw (2005) est incontournable pour bien cerner la complexité des enjeux et comprendre comment les contextes normatif et légal ont le potentiel de marginaliser des personnes ou des groupes en fonction de leurs identités.

\section{Les valeurs personnelles, professionnelles et sociales}

Au-delà des normes prescrites et communément acceptées, les stagiaires doivent aussi réfléchir à leurs valeurs personnelles, professionnelles ou sociales que le dilemme éthique interpelle. La prise en compte de ces dimensions pour les autres personnes touchées, comme dans l'exemple de la fessée, est tout aussi nécessaire. Ici, la justice sociale, la solidarité, la protection des enfants et le respect de la dignité humaine constituent des valeurs qui sont susceptibles d'intervenir. Le fait d'avoir vécu ou d'avoir été exposée à des injustices peut aiguiser la sensibilité de la stagiaire, colorant ainsi ses valeurs, la rendant davantage protectrice. Quoi qu'il en soit, ces valeurs agissant dans la situation doivent être remises en question en fonction des positions de privilège ou d'oppression de la stagiaire et des autres personnes impliquées (Bilge, 2010, 2009). Des valeurs personnelles demeurent bien souvent ignorées par les stagiaires jusqu'au moment d'entrer en contact avec celles embrassées par les citoyens du pays hôte (Barlow, 2007). 


\section{Les conséquences positives et négatives sur soi, sur les autres et sur l'environnement}

"...les stagiaires
auront à réfléchir,
à identifier et à
évaluer l'ensemble
des conséquences
positives et négatives
sur soi, sur les autres
et sur l'environnement,
notamment la
communauté et les
institutions. "

"...les stagiaires auront à réfléchir, à identifier et à évaluer l'ensemble des conséquences positives et négatives sur soi, sur les autres et sur l'environnement, institutions. "
Les options de résolution du dilemme sont essentiellement portées par des valeurs qui se confrontent devant la conduite à tenir. Or, pour chacune des options, les stagiaires auront à réfléchir, à identifier et à évaluer l'ensemble des conséquences positives et négatives sur soi, sur les autres et sur l'environnement, notamment la communauté et les institutions. La prise en compte de la portée de ces conséquences, leurs implications à court et à long terme et la probabilité qu'elles se manifestent sont toutes des dimensions à explorer dans l'exercice de reconnaissance des conséquences (Legault, 1999). Par ailleurs, la réflexion sur les systèmes de privilège et d'oppression, les identités, les contextes et les expériences vécues est fondamentale dans l'évaluation des conséquences. Sur le plan contextuel, dans notre exemple de la punition corporelle imposée aux deux enfants, la stagiaire peut mettre à risque leur sécurité en intervenant pour les protéger, la sécurité d'emploi d'un collègue peut être aussi menacée et l'octroi de fonds à l'institution peut être remis en cause parce que l'organisme subventionnaire n'entérine pas des pratiques comme la fessée. Les conséquences potentielles varieront en fonction de l'accès ou non à d'autres ressources de placement pour l'enfant, de la disponibilité ou non d'emplois, ou des sources possibles de financement.

\section{La décision et son évaluation (raisonnement conséquentialiste ou déontologique)}

Au terme de l'analyse de l'ensemble des éléments qui ont été soulevés, les stagiaires devront se positionner et prendre la meilleure décision possible dans les circonstances. Cependant, l'importance accordée aux divers éléments varie en fonction de la personne qui décide (Albert, 2006), c'est-à-dire sa trajectoire 
personnelle, ses identités et ses expériences (Crenshaw, 2005). Les stagiaires, dans ces circonstances, ont la possibilité d'attribuer plus de poids aux conséquences ou aux normes. Dans notre exemple, même si elle est fortement interpellée par les conséquences de la fessée sur les enfants et qu'elle est prête à intervenir pour les protéger, la stagiaire pourrait tout aussi bien choisir de se conformer aux normes de l'institution et ainsi ne pas intervenir en raison de son statut ou de son insécurité devant un contexte culturel, social et politique qui n'est pas le sien.

\section{La réflexion critique et dialogique sur le « caractère universalisable des raisons d'agir " (Legault, 1999, p. 277), soit l'impartialité, la réciprocité et l'exemplarité}

"...cette réflexion exige une ouverture culturelle et doit se prêter à un exercice propice à un renversement éventuel de perspective. Ce regard sur soi et sur la situation peut se réaliser en intégrant les principes associés à l'analyse intersectionnelle, c'est-àdire, un questionnement sur sa position de privilège et d'oppression, ses identités, ses contextes de vie et ses expériences.»
Quand la décision est prise, il importe de l'évaluer et idéalement d'en discuter avec les principaux intéressés, sinon avec un collègue, un superviseur ou une personne qui agit comme consultant pour le stage. Legault $(1999$, p. 277) propose une grille de réflexion qui permet de mettre en perspective la décision en fonction du caractère universalisable des raisons qui ont conduit à la décision. C'est à partir de trois critères (l'impartialité, la réciprocité et l'exemplarité) que cet exercice est réalisé. Les stagiaires doivent évaluer leur décision en s'interrogeant sur son impartialité, en se demandant par exemple si un jury impartial serait convaincu des raisons menant à une telle décision. Ainsi, notre stagiaire pourrait évaluer la justesse de l'argumentaire qui a conduit à sa décision face à la punition corporelle infligée aux enfants en s'imaginant présenter ses raisons d'agir devant un comité de l'Organisation des Nations unies responsable de l'application de la Convention relative aux droits de l'enfant. Les stagiaires doivent également se pencher sur le critère de réciprocité en se demandant si, du point de vue de la personne qui perd le plus dans la situation, leur décision demeurerait la même. L'évaluation de ce critère et l'identification de la personne qui a le plus à perdre dans cette 
situation doivent être filtrées par les systèmes d'oppression et de privilège dans le contexte du pays d'accueil. Enfin, les stagiaires ont à se questionner à savoir en quoi, dans une situation semblable, leur décision pourrait servir d'exemple dans le contexte social, politique et culturel du pays d'accueil. Il est à noter cependant que le fait de se poser ces questions, au-delà des réponses qu'on puisse leur donner, permet essentiellement de jeter un regard sur la décision avec une distanciation qui favorise l'adoption d'une autre perspective. Mais cette réflexion exige une ouverture culturelle et doit se prêter à un exercice propice à un renversement éventuel de perspective. Ce regard sur soi et sur la situation peut se réaliser en intégrant les principes associés à l'analyse intersectionnelle, c'est-à-dire, un questionnement sur sa position de privilège et d'oppression, ses identités, ses contextes de vie et ses expériences.

\section{Conclusion}

La contribution du modèle de délibération éthique proposé ici réside dans le fait qu'il intègre l'analyse intersectionnelle. En ce sens, il rend visible des dimensions souvent occultées, permettant de mettre en lumière des réalités complexes, tout en offrant l'occasion de saisir l'aspect unique des situations qui se présentent lors d'un stage à l'international. Bien que les stagiaires doivent tout au long de leur expérience demeurer sensibles aux valeurs culturelles et sociales privilégiées par leurs pays d'accueil, ce modèle leur permet de réfléchir à leur positionnement tout en leur donnant la possibilité d'éviter le piège du relativisme culturel ou de l'ethnocentrisme.

Il reste cependant qu'un des défis associés aux dilemmes éthiques rencontrés dans des contextes de stages internationaux se situe dans l'appréhension du malaise qui déclenche le constat qu'on est devant un tel dilemme. En effet, en présence d'un choc culturel, souvent vécu de façon inconsciente, la perception du malaise peut en être affectée. Dubois et Ntetu (2000) décrivent le choc culturel comme un processus suscitant des tensions lorsque des personnes explorent leurs différences sur le plan des attitudes 
"Le stage

international quand

il est bien encadré

constitue un laboratoire

d'apprentissage

pertinent qui favorise

l'approfondissement

de la connaissance de

soi, de la conscience

de l'Autre et de la

réflexion critique.

D'ailleurs, les stagiaires de l'ÉTS reconnaissent à leur retour avoir vécu une transformation personnelle qui risque d'avoir un impact sur leur trajectoire professionnelle. » ou des comportements. Ces tensions peuvent provoquer, entre autres, un sentiment de dépassement, de déstabilisation, la perte de points de repère embrouillant ainsi le processus de réflexion. Cela soulève dès lors des enjeux dont les personnes responsables des stages internationaux doivent prendre en compte dans le processus d'accompagnement des stagiaires.

Il est essentiel d'offrir aux stagiaires des sessions de formation qui permettent d'exposer ce modèle dans une formule offrant l'occasion de le mettre en application à l'aide de cas fictifs autour desquels il leur est possible de délibérer. Par ailleurs, la qualité de la relation développée entre les responsables des stages et les stagiaires qui partent à l'étranger est fondamentale afin de leur permettre d'exprimer les malaises ressentis et par conséquent les dilemmes rencontrés. La relation étant à elle seule insuffisante, les stagiaires doivent avoir dans leur coffre des outils, notamment un journal de bord et des fiches réflexives, leur permettant de prendre conscience des malaises et de la présence de dilemmes éthiques. Selon Payne et Askeland (2008), il est impossible d'apprendre de la différence sans un processus de réflexion critique. De surcroît, Nuttman-Shwartz et Berger (2012) parlent de la nécessité d'un accompagnement serré, qui se traduit à l'ÉTS par un suivi à distance régulier pour encadrer de telles expériences. Au retour, il n'en reste pas moins que les stagiaires peuvent revenir avec des situations non résolues ou des malaises persistants. Pour Tiessen et Kumar (2013), le debriefing permet de discuter de situations problématiques rencontrées et de donner sens à ce qui a été vécu. Ainsi, le recul, à la fois de distance et de temps, est parfois requis pour parvenir à la disposition nécessaire à l'évaluation de dilemmes.

Le stage international quand il est bien encadré constitue un laboratoire d'apprentissage pertinent qui favorise l'approfondissement de la connaissance de soi, de la conscience de l'Autre et de la réflexion critique. D'ailleurs, les stagiaires de l'ÉTS reconnaissent à leur retour avoir vécu une transformation personnelle qui risque d'avoir un impact sur leur trajectoire professionnelle. Cependant, la crainte récurrente pour plusieurs est celle d'une préparation inadéquate pour le marché du travail 
canadien. Green, et collab. (2008) soulèvent des préoccupations et des peurs similaires de la part d'étudiantes ou d'étudiants effectuant un séjour d'études dans un autre pays, à savoir que leurs expériences ne sont pas les mêmes que celles que vivent leurs collègues au Canada. L'expérience des quelque vingt-cinq dernières années de stages internationaux à l'ÉTS nous permet d'observer que la préparation à intégrer un nouvel emploi en travail social pour les stagiaires à l'international est semblable à celle d'étudiantes ou étudiants qui auraient effectué leur stage en contexte canadien, mais dans un milieu différent de celui de l'emploi décroché. La clé dans la transition entre le stage et l'emploi réside dans la transférabilité des apprentissages.

Telle que proposée dans le modèle, l'analyse intersectionnelle adaptée à la délibération éthique est applicable à la fois dans un contexte national et international. En effet, bien que l'interculturel soit plus évidemment observé dans un rapport à l'Autre qui soit empreint de différences ethniques, linguistiques ou culturelles, il est également présent dans un rapport à un Autre avec lequel on entretient davantage de proximité. Marsiglia et Kulis (2009, p. 4 [notre traduction]) précisent : «Bien qu'une communauté puisse partager une identité culturelle commune, ses membres ont des façons uniques d'exprimer cette identité collective. La classe sociale, la race, l'ethnicité, la religion et la langue contribuent de façon importante à l'identité culturelle des individus.» À la lumière de ce constat, nous considérons que ce modèle est pertinent, peu importe les milieux dans lesquels les stagiaires réalisent leur formation pratique. En effet, étant donné l'essence même du travail social et le fait qu'il doive intervenir, entre autres, aux frontières de rapports d'oppression, de situations d'injustice et d'expériences singulières dans des contextes pluriels, la réflexivité à laquelle nous convie ce modèle prend tout son sens.

\section{Bibliographie}

ASSOCIATION CANADIENNE POUR LA FORMATION EN TRAVAIL SOCIAL (2013).

Normes d'agrément, Approuvé par le Conseil d'administration, juin 2013.

ALBERT, Hélène (2006). Les dilemmes éthiques des travailleuses sociales francophones auvrant dans le domaine de la protection de l'enfance au Nouveau-Brunswick [Thèse de doctorat], Québec, Faculté des sciences sociales, Université Laval. 
BARLOW, Constance A. (2007). "In the third space: A case study of Canadian students in a social work practicum in India ", International Social Work,Vol. 50, N², p. 243-254.

BECK, Philip (2003). "Impressions from an English exchange student in Canada ", dans Lena DOMINELLI et Wanda THOMAS BERNARD (Dirs.), Broadening horizons: International exchanges in social work, Aldershot, Ashgate Publishing Limited, p. 129-134.

BELL, Karen, et A.W. (Bill) ANSCOMBE (2013). "International field experience in social work: Outcomes of a short-term study abroad programme to India ", Social Work Education: The International Journal, Vol. 32, $\mathrm{N}^{\circ}$ 8, p. 1032-1047.

BILGE, Sirma (2009). "Théorisation féministe de l'intersectionalité », Diogène, N²25, p. 70-88.

BILGE, Sirma (2010). "De l'analogie à l'articulation : théoriser la différence sociale et l'inégalité complexe ", dans Elise PALOMARES et Armelle TESTENOIRE (Dirs.), Prismes féministes. Qu'est-ce que l'intersectionnalité?, Paris, L'Harmattan, p. 43-64.

COMBAHEE RIVER COLLECTIVE (2008). "Déclaration du Combahee River Collective ", dans Elsa DORLIN (Dir.), Black feminism. Antologie du féminisme africain-américan, 1975-2000, Paris, L'Harmattan, p. 59-73.

CRENSHAW, Kimberly Williams (2005). « Cartographie des marges : intersectionnalité, politique de l'identité et violences contre les femmes de couleur ", Cahiers du genre, № 39, p. 51-82.

DAS, Chaitali, et Janet CARTER ANAND (2012). «Strategies for critical reflection in international contexts for social work students ", International Social Work, juin, p. 1-16. En ligne : http:// isw. sagepub.com/content/early/2012/06/13/0020872812443693.full.pdf+html

DUBOIS, Michel, et Antoine-L. NTETU (2000). « Learning cultural adaptation through international social work training ", Canadian Social Work,Vol. 2, N 2, p. 41-52.

ENGSTROM, David, et Loring P.JONES (2007). «A Broadened horizon:The value of international social work internships ", Social Work Education:The International Journal, Vol. 26, № 2, p. 136-150.

EPPRECHT, Marc (2004). "Work-study abroad courses in international development studies: Some ethical and pedagogical issues ", Revue canadienne d'études du développement, Vol. XXV, $\mathrm{N}^{\circ} 4$, p. 687-706.

FÉDÉRATION INTERNATIONALE DES TRAVAILLEURS SOCIAUX ET ASSOCIATION INTERNATIONALE DES ÉCOLES DE TRAVAIL SOCIAL (2004). Ethics in social work, statement of principles, Berne, Suisse.

GREEN, Barbara F., et collab. (2008). « Studying abroad: a multiple case study of nursing students' international experiences ", Nurse Education Today, $N^{\circ} 28$, p. 981-992.

HERON, Barbara (2005). « Changes and challenges: Preparing social work students for practicums in today's Sub-Saharan African context », International Social Work, Vol. 48, N 6, p. 782-793.

HILL COLLINS, Patricia (1990). Black feminist thought: Knowledge, consciousness, and the politics of empowerment, Boston, Unwin Hyman, 384 p.

hooks, bell (2008). "Sororité : la solidarité politique entre les femmes », dans Elsa DORLIN (Dir.), Black feminism. Antologie du féminisme africain-américan, 1975-2000, Paris, L'Harmattan, p. 113-134.

HULKO, Wendy (2009). "The time- and context-contingent nature of intersectionaly and interlocking oppressions ", Affilia, Vol. 24, № 1, p. 44-55.

LAMOUREUX, Henri (2003). Éthique, travail social et action communautaire, Québec, Presses de l’Université du Québec, 268 p. 
LANTEIGNE, Isabel, et Lise SAVOIE (2010). «Le confort et l'indifférence. L'enseignement féministe à l'ère de l'individualisme ", Revue canadienne de service social,Vol. 27, № 2, p. 171-185.

LEGAULT, Georges (1999). Professionnalisme et délibération éthique, Québec, Presses de l'Université du Québec, 306 p.

MARCHAND, Isabelle, et Sandrine RICCI (2010). «Sexisme et racisme : la diversité ethnoculturelle, défi au mouvement féministe ", dans Christine CORBEIL et Isabelle MARCHAND (Dirs.), L'intervention féministe d'hier à aujourd'hui. Portrait d'une pratique sociale diversifiée, Montréal, Les Éditions du Remue-Ménage, p. 65-92.

MATHIESEN, Sally G., et LAGER, Patricia (2007). "A model for developing student exchanges ", Social Work Education : International Journal,Vol. 26, № 3, p. 280-291.

MATTISON, Marian (2000). "Ethical decision making: The person in the process ", Social Work, Vol. 45, N³, p. 201-212.

MARSIGLIA, Flavio Francisco, et Stephen KULIS (2009). Culturally grounded social work. Diversity, oppression, and change, Chicago, Illinois, Lyceum Books, Inc, 400 p.

NOBLE, Carolyn (2003). " “What am I doing here, really?” - Students' and teachers' reflections on international placements ", dans Lena DOMINELLI et Wanda THOMAS BERNARD (Dirs.), Broadening horizons: International exchanges in social work, Aldershot, Ashgate Publishing Limited, p. 257-276.

NUTTMAN-SHWARTZ, Orit, et Roni BER GER (2012). « Field education in international social work: Where we are and where we should go ", International Social Work, Vol. 55, No 2, p. 225-243.

ORGANISATION DES NATIONS UNIES (1989). Convention relative aux droits de l'enfant, réf. du 18 mars 2014, http://www.ohchr.org/FR/ProfessionalInterest/Pages/CRC.aspx

PAYNE, Malcolm, et Gurid Aga ASKELAND (2008). Globalization and international social work. Postmodern change and challenge, Aldershot, Ashgate Publishing Limited, $188 \mathrm{p}$.

QUINTIN, Jacques, et Anne-Marie BOIRE-LAVIGNE (2013). "Exercer une délibération en éthique clinique avec le souci de l'expérience des personnes ", Cahier Professeur Éthique Profession MD III 2013, Sherbrooke, Université de Sherbrooke.

RANKOPO, Morena J., et Kwaku OSEI-HWEDIE (2011). « Globalization and culturally relevant social work: African perspectives on indigenization ", International Social Work, Vol. 54, $\mathrm{N}^{\circ} 1$, p. 137-147.

RAZACK, Narda (2002). «A critical examination of international student exchanges », International social work, Vol. 45, N², p. 251-265.

REAMER, Frederic G. (1995). Social work values and ethics, New York, Columbia University Press, $240 \mathrm{p}$.

SEWPAUL, Vishantie (2003). "Reframing Epistemologies and Practices through International Exchanges: Global and Local Discourses in the Development of Critical Consciousness ", dans Lena DOMINELLI et Wanda THOMAS BERNARD (Dirs.), Broadening horizons: International exchanges in social work, Aldershot, Ashgate Publishing Limited, p. 297-332.

SHIELDS, Stephanie A. (2008). "Gender: An intersectionality perspective ", Sex Roles, Vol. 59, p. 301-311. 
TIESSEN, Rebecca, et Paritosh KUMAR (2013). «Ethical challenges encountered on Learning/ Volunteer abroad programmes for students in international development studies in Canada: Youth perspectives and educator insights ", Revue canadienne d'études du développement, Vol. 43, $N^{\circ} 3$, p. 416-430.

WELDON, Laurel S. (2006). "The Structure of intersectionality: A comparative politics of gender », Politics \& Gender, Vol. 2, N 2, p. 235-248. 